This item was submitted to Loughborough's Research Repository by the author.

Items in Figshare are protected by copyright, with all rights reserved, unless otherwise indicated.

\title{
Integrating accessibility and functional requirements
}

PLEASE CITE THE PUBLISHED VERSION

PUBLISHER

(c) Springer Verlag Berlin Heidelberg

VERSION

AM (Accepted Manuscript)

LICENCE

CC BY-NC-ND 4.0

REPOSITORY RECORD

Baguma, Rehema, R.G. Stone, Jude T. Lubega, and Th.P. van der Weide. 2019. "Integrating Accessibility and Functional Requirements". figshare. https://hdl.handle.net/2134/6100. 
This item was submitted to Loughborough's Institutional Repository (https://dspace.lboro.ac.uk/) by the author and is made available under the following Creative Commons Licence conditions.

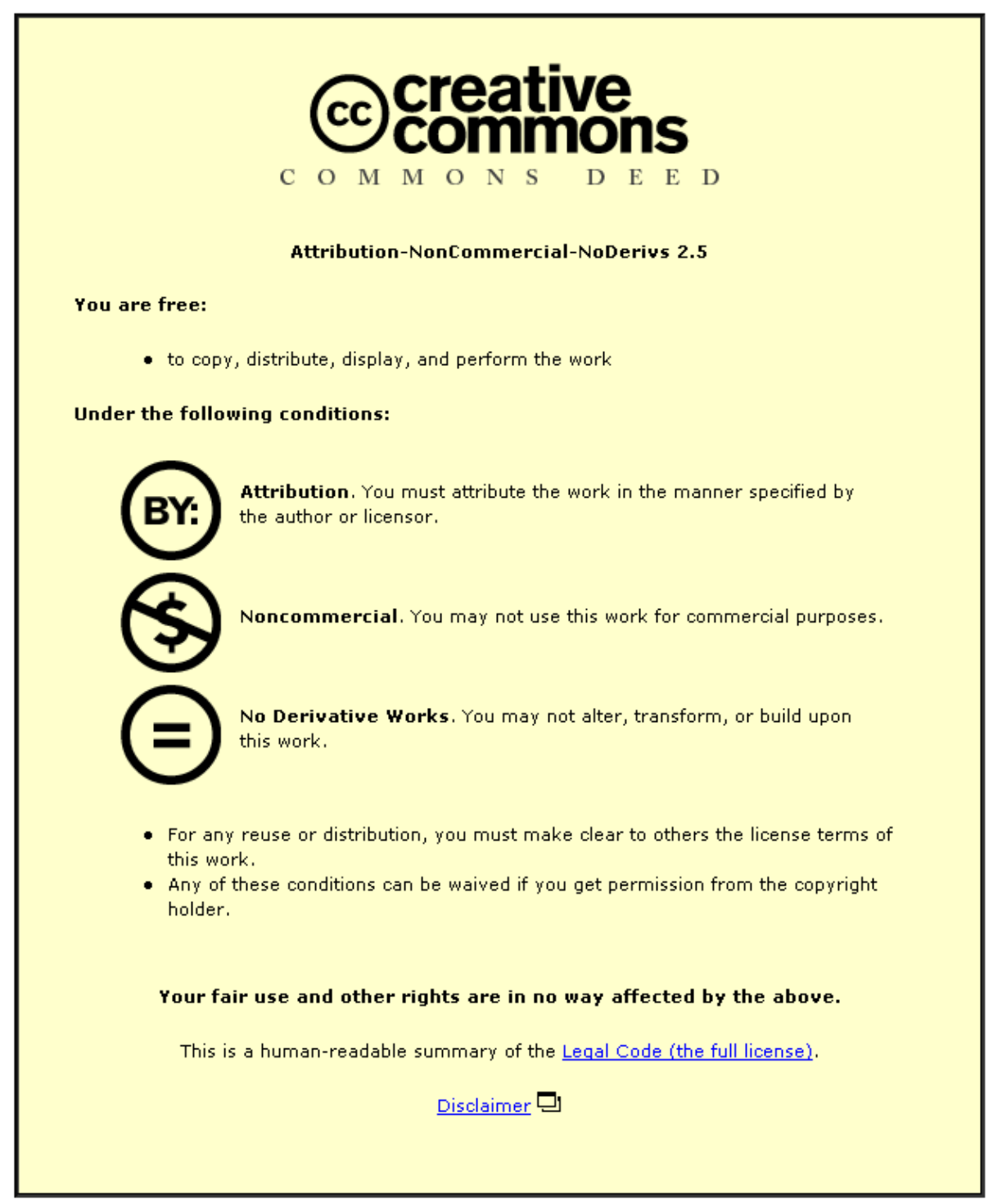

For the full text of this licence, please go to: http://creativecommons.org/licenses/by-nc-nd/2.5/ 


\title{
Integrating Accessibility and Functional Requirements
}

\author{
Rehema Baguma ${ }^{1}$, Roger G. Stone ${ }^{2}$, Jude T. Lubega ${ }^{1}$, Th.P. van der Weide ${ }^{3}$ \\ ${ }^{1}$ Faculty of Computing \& IT, Makerere University, P. O. Box, 7062, Kampala \\ ${ }^{2}$ Loghborough University, Loughborough, LE11 3TU, UK \\ ${ }^{3}$ Radboud University, PO Box 9010, NL-6500 ED Nijmegen, The Netherlands \\ rbaguma@cit.mak.ac.ug, R.G.Stone@lboro.ac.uk, jlubega@cit.mak.ac.ug, \\ Th.P.vanderWeide@cs.ru.nl
}

\begin{abstract}
Initial research on Web accessibility was focused on testing completed Web pages. More recently, the focus is moving to integrating accessibility features into coding tools such as Dreamweaver 8 and plugins notably LIFT. Thus accessibility is being considered slightly earlier in the development process. However, the state of Web accessibility is still disappointing even on websites that have followed the guidelines and or used evaluation and coding tools. We are proposing an approach to start considering accessibility much earlier. Our purpose is to address accessibility in the context of what is to be done and who will be participating. In this paper, we present views of Web developers about this approach. We then show (using a case study) how Web developers can elicit accessibility requirements alongside functional requirements and integrate the two to obtain conceptual models with explicit traces of accessibility requirements integrated with functional requirements. Finally we discuss lessons learnt from the case study and common benefits of the approach for Web accessibility and Web projects.
\end{abstract}

Keywords: Web accessibility, Web accessibility requirements, functional requirements, non functional requirements

\section{Introduction}

An accessible website is one that is sufficiently flexible to be used by all people including People with Disabilities (PWDs). Although accessibility is a vital quality attribute for PWDs, it has not yet gained much recognition as a crucial non-functional requirement like security, performance, accuracy and usability [1, 2, 3].

Initial research on Web accessibility was focused on testing completed Web pages using automated tools like Web Accessibility Verifier. But automated evaluation tools are technical oriented with less focus on usability. As a result, passing automated accessibility tests does not mean a website is accessible [10,11]. An image may pass the test because it has an alternative text description but the image can still be inaccessible if the alternative text does not make sense to the user.

More recently, the focus is moving to integrating accessibility features into coding tools (such as Dreamweaver 8) and plugins notably LIFT, a plugin for Dreamweaver and Microsoft front page. Thus accessibility is being considered slightly earlier in the development process. 
However, the rate of Web accessibility is still disappointing even on websites that have followed accessibility guidelines and or used the evaluation and coding tools [4], $[5,6,7], 8,9]$. Common accessibility problems such as inappropriate 'alt' text and broken skip navigation links are caused by focusing on technical aspects at the expense of human aspects of computing [10].

We are proposing an approach to integrate accessibility into requirements analysis and specification activities. The purpose of this approach is to address accessibility in the context of what is to be done and who will be participating. We present views of a handful of Web developers (involved in developing accessible websites) about the concept. We then show how Web developers can elicit accessibility requirements alongside functional requirements and integrate the two to obtain conceptual models with explicit traces of accessibility requirements. This is supported by a case study. Finally we discuss lessons learnt from the case study and common benefits of the approach to Web accessibility and Web projects.

\section{Views of Web Developers about Integrating Accessibility into Requirements Analysis and Specification}

\subsection{Methodology}

A survey questionnaire was sent to the British Computer Association of the Blind (BCAB) mailing list, a mailing list for computing professionals involved in accessibility and computer users with disabilities mainly visual disabilities in Britain. The survey targeted Web developers involved in developing accessible websites. It covered: the time spent on different stages of Web development, what stage accessibility is considered and why, if addressing accessibility during requirements analysis and specification would appeal to them and why, and any other comments.

\subsection{Response Rate}

The respondents included: 3 Web developers, 2 software developers, 1 content manager and 1 advanced user interested in developing accessible websites. But, we only considered 5 developers and 1 content manager.

\subsection{Time Spent on Different Stages of Web Development}

Table 1. Time spent on different stages of Web development

\begin{tabular}{|l|l|l|l|l|l|l|l|}
\hline \multicolumn{1}{|c|}{ Stage } & \multicolumn{5}{c|}{ Time (\%) } & Average (\%) \\
\hline & Dev1 & Dev2 & Dev3 & Dev4 & Dev5 & Dev6 & \\
\hline Analysis \& specification & 10 & 30 & 5 & 10 & 55 & 50 & 27 \\
\hline Coding & 70 & 50 & 90 & 70 & 35 & 50 & 61 \\
\hline Testing & 20 & 20 & 5 & 20 & 10 & 50 & 21 \\
\hline
\end{tabular}


As shown in table 1, developers spend more time on coding followed by requirements analysis and specification and the least time on testing.

\subsection{Stage where Accessibility is considered}

Table 2. Stage where accessibility is considered

\begin{tabular}{|l|l|l|}
\hline \multicolumn{1}{|c|}{ Stage } & \multicolumn{2}{|c|}{ Response } \\
\hline & Yes & No \\
\hline Requirements analysis \& specification & 4 & 1 \\
\hline Coding & 5 & 0 \\
\hline Testing & 5 & 0 \\
\hline
\end{tabular}

Note: The content manager skipped this question since his role does not involve developing websites.

As shown in table 2, some developers don't consider accessibility during requirements analysis and specification.

\subsection{The reason for the practice about which stage to consider accessibility}

Participants gave the following reasons:

- I write assistive technologies for blind people

- Accessibility is a highly important factor. Designing from the outset makes the project progress more smoothly.

\subsection{If addressing accessibility during analysis and specification is appealing?}

Five participants said yes, and one said no. Those who said yes gave the following reasons:

- If a fairly robust methodology is presented to me. Trying to communicate accessibility on a technical level to the client can be a difficult process. Referring to legislation is normally the best way to do this. It is hard to communicate accessibility to the client on a technical level. But at requirements level, it is easier.

- I already do so

- I think you have to do this as fundamentally, the whole design is based around accessibility and excellent usability

\subsection{Other Comments}

Participants gave the following comments:

- Web accessibility is an interesting topic e.g. many guidelines say that you should have high contrast sites e.g. yellow on black or black on white but many people with reading but not vision impairments such as dyslexia need low contrast sites and many old people find that yellow on black halates. Different groups also have different levels of political power. Therefore it is important to know one's audience. 
- Accessibility is something that can be difficult to design from the beginning of the design process. Rapidly advancing technology and practice in this area can make accessibility something that is often distinct from the rest of the Web design process. A robust methodology on designing for accessibility and usability would be a welcome resource.

\subsection{Discussion}

From the results presented in 2.3 to 2.7 , some Web developers involved in developing accessible websites consider accessibility during requirements gathering and specification and others don't. But also little time is generally spent on this activity compared to coding. Therefore it is possible that accessibility needs don't get enough attention. This could be one of the reasons for persistent low levels of Web accessibility. Therefore, there is need for developers to give accessibility more attention during requirements analysis and specification to increase usability of developed websites for people with disabilities.

The results in 2.5 to 2.7 also show that Web developers involved in developing accessible websites believe that integrating accessibility into requirements analysis and specification has a potential to improve accessibility of websites and make the project progress more smoothly. Hence a methodology on designing for accessibility and usability is a welcome resource.

\section{Integrating Accessibility needs into Requirements Analysis and Specification}

\subsection{Integrating Accessibility into Requirements Analysis}

A requirement is a software capability that must be met or possessed by a system or a system component to satisfy a contract, standard, or desired need [14]. Requirements are classified into functional and non-functional requirements. Functional requirements present a complete description of how the system will function from the users' perspective [14]. On the other hand, non-functional requirements dictate properties and impose constraints on the system [14]. They specify attributes the system should have, rather than what the system will do e.g. security, reliability, usability and accessibility. Although considerable research has been done on how to capture and specify common non-functional requirements such as security, performance, usability like in [3], [1], [2], not much has been done about accessibility.

In this sub section, we show how accessibility requirements can be analyzed together with functional and other non functional requirements using User Centered Design (UCD) techniques [12]. The focus of UCD is to produce usable systems. Our choice of UCD techniques was motivated by the fact that accessibility is a subset of usability [12], a mechanism of making systems work for the user [5]. 
Henry [12] believes that in practice, accessibility design techniques do fit well into established UCD processes. But Web designers need to include the widest range of users and situations in the UCD process. To provide practical guidance, Henry provides a checklist of points at which accessibility can be addressed in the UCD process namely:

- Business and usability goals should include meeting accessibility requirements

- Understanding user characteristics should include users with various disabilities

- Environmental aspects should include disability and limiting situations e.g. visual limitations, hands-free, noisy, use of Assistive Technologies or mobile devices

- Workflow scenarios should include use of an assistive technology

- Usability testing should include participants with disabilities

The above points belong to the different phases of the UCD approach namely: analysis, design and evaluation. In this section, we focus on the analysis.Design is covered in the next section.

Analysis or user analysis is the stage where details about who uses or is to use the system (roles, characteristics such as knowledge, experience, and skill with similar systems, environment; frequency of use, and depending on the type of system, their hardware, software, and assistive technologies) are collected [12]. Results of the analysis are documented in user group profiles, personas and scenarios. A user group profile describes characteristics of users of a system or product e.g. demographics, responsibility, hardware \& software environment etc. A persona is a fictional characterization of a user that is aimed at making the users seem more real to help designers keep realistic ideas of users throughout the design process [12]. While a scenario is a description of a persona using a product to achieve a goal.

User group profiles, personas and scenarios can be used to put into context, the application of Web accessibility guidelines e.g. WCAG according to the needs of users including users with disabilities. The Web developer can apply the guidelines based on the characteristics and environment of users including users with various disabilities. Such an approach caters for both technical and user interface accessibility of developed websites. Henry [12] provides detailed guidance on how to include accessibility considerations in user group profiles, personas and scenarios.

The next sub section discuss how to integrate accessibility requirements obtained during requirements analysis into requirements specification models such as use cases, scenarios and class diagrams.

\subsection{Integrating Accessibility into Requirements Specification.}

According to McEwen [14], non-functional requirements (NFRs) are not always in the front of stakeholders' minds, and analysts must make a special effort to draw them out. In addition, NFRs are always informally stated, often contradictory, difficult to enforce during development and difficult to evaluate for the customer prior to delivery [14]. Due to these challenges, Ceysneiros and Leite [14] recommend presenting NFRs in NFR goal graphs to obtain detailed reasoning and resolve any conflicts. We use Ceysneiros and Leite's NFR goal graphs approach (modified to suite our goal) to obtain detailed reasoning for accessibility requirements. We call our goal graphs accessibility requirements (AR) graphs. In the AR graphs, accessibility 
requirements are presented as goals (roots of an AND graph) that are decomposed into sub goals until all the necessary accessibility design considerations are represented at the leaf levels of the graphs. Generally, developing a goal graph starts from a very high level goal e.g. separate content from presentation and refines it into more specific ways of achieving this goal, e.g. use HTML mark up to describe document structure and CSS to define presentation. This process continues until the level where the defined action (s) will be sufficient to implement the goal.

Each AR goal graph has a subject matter presented at the root of the graph. This links the AR graph to the concerned functional requirement or business object. The accessibility considerations for a functional requirement are presented according to the interactive tasks between the user and the system. This is because accessibility is a sub set of usability [12]. For example if the business object is application form, then accessibility requirements have to be defined for all the tasks involved in accessing and filling the application form.

After representing accessibility requirements in AR graphs, the next step is to integrate the accessibility requirements into functional requirements specification models such as use cases, class diagrams and scenarios. Due to space limitations, we only cover use cases. But for scenarios, collaboration diagrams and class diagrams, a similar approach to the one used for use cases is followed.

To integrate accessibility requirements into use cases , for every use case, check the AR graph for tasks associated with the use case. For every task associated with the use case, add a new use case as an include link connected to the functional requirement use case. Every use case included to meet accessibility requirements must be named in the format: \{AR_topic [Accessibility]\} e.g. provide for keyboard acess[accessibility]. The aim of this expression is to add traceability between functional perspectives and accessibility perspectives. This traceability link helps to show that the use cases are there to make the functional tasks accessible.

\subsection{Case Study: Online Module Review System for the Department of Computer Science, Loughborough University}

We tested the approach on one project that is the development of an online module review system for the department of computer science, Loughborough University. The module review system is used to review modules taught in the department. At the time of the case study, the department was in the process of changing from an offline to an online system. We got a chance to participate in requirements analysis and specification particularly to integrate accessibility needs. To achieve this, we first analyzed the offline system (form) and later held a discussion with the Quality Manager who was also the one to develop the proposed online system. From the analysis and discussion, we obtained information about the current and the proposed system namely; users and their tasks, logical structure of the proposed system, features of the module review system that might affect Web accessibility and security requirements. Based on this information, a checklist of Web accessibility requirements for the proposed system was developed. The accessibility requirements were precisely presented in Accessibility Requirements (AR) graphs according to the expected interactions between the users and the system. The expected interactions 
included; open review system, read review, comment, and submit review. Figure 1 is the AR graph that shows a break down of accessibility considerations necessary to make tasks involved in using the online module review system accessible to all users including those with disabilities.

Figure 1 shows that for a member of staff who wants to review a given module, a number of tasks are involved namely: open review system, read review form, comment and submit review. All these tasks must be accessible to the user hence the expression Accessibility[task name] for each task. Under each task there is an AND node with all the required accessibility considerations. For general website projects that serve different business needs, AR graphs have to be developed for all topics of the functional needs.

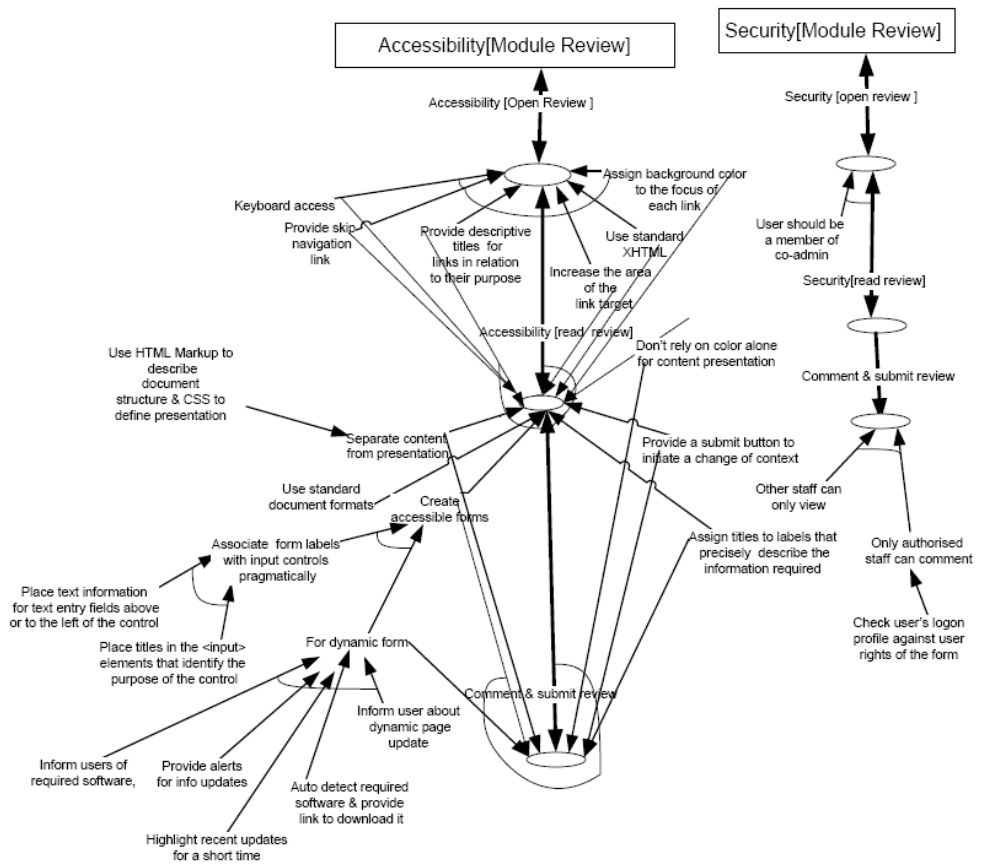

Fig. 1. shows the AR graph for tasks on the online module review system

In addition to the accessibility requirements, figure 1also shows that the project has security requirements that is access to the system is restricted to members of the coadmin. More so, even among members of co-admin, only those authorized can comment on it. Others can only view.

After a detailed reasoning of required accessibility considerations, we integrated the accessibility requirements into the use case diagram of the module review system. The module review system had six actors. The internal examiner comments on; formal feedback, informal feedback, module results, previous action plan, gives general comments and sets the action plan for next academic year. The external 
examiner, other examiners and moderator comment on the module. The quality manager approves module evaluation. Other staff can view module evaluation.

To integrate accessibility requirements into the use case diagram, for every use case, we checked the AR graph for tasks associated with the use case e.g. does comments on formal feedback involve opening the review form? For every accessibility requirement associated with a given use case, we added a new use case as an include link connected to the use case. Every use case included to meet accessibility requirements was named in the format: \{AR_topic [Accessibility] to add traceability between functional and accessibility perspectives.

Figure 2 shows the use case diagram of the module review system after adding accessibility requirements that are necessary to make the system accessible. The required accessibility considerations are added as include use cases since they are/can be used by many other use cases.

Note: The rectangle on 'comments on formal feedback' use case was used to simplify presentation. The include links starting from the rectangle mean that other than being linked to the 'comments on formal feedback' use case, they are also all 'housed' collectively in the rectangle hence any reference to one of them can be done by linking to the rectangle. This is what is done to present required accessibility considerations for the internal examiner, external examiner, other examiners and moderator.

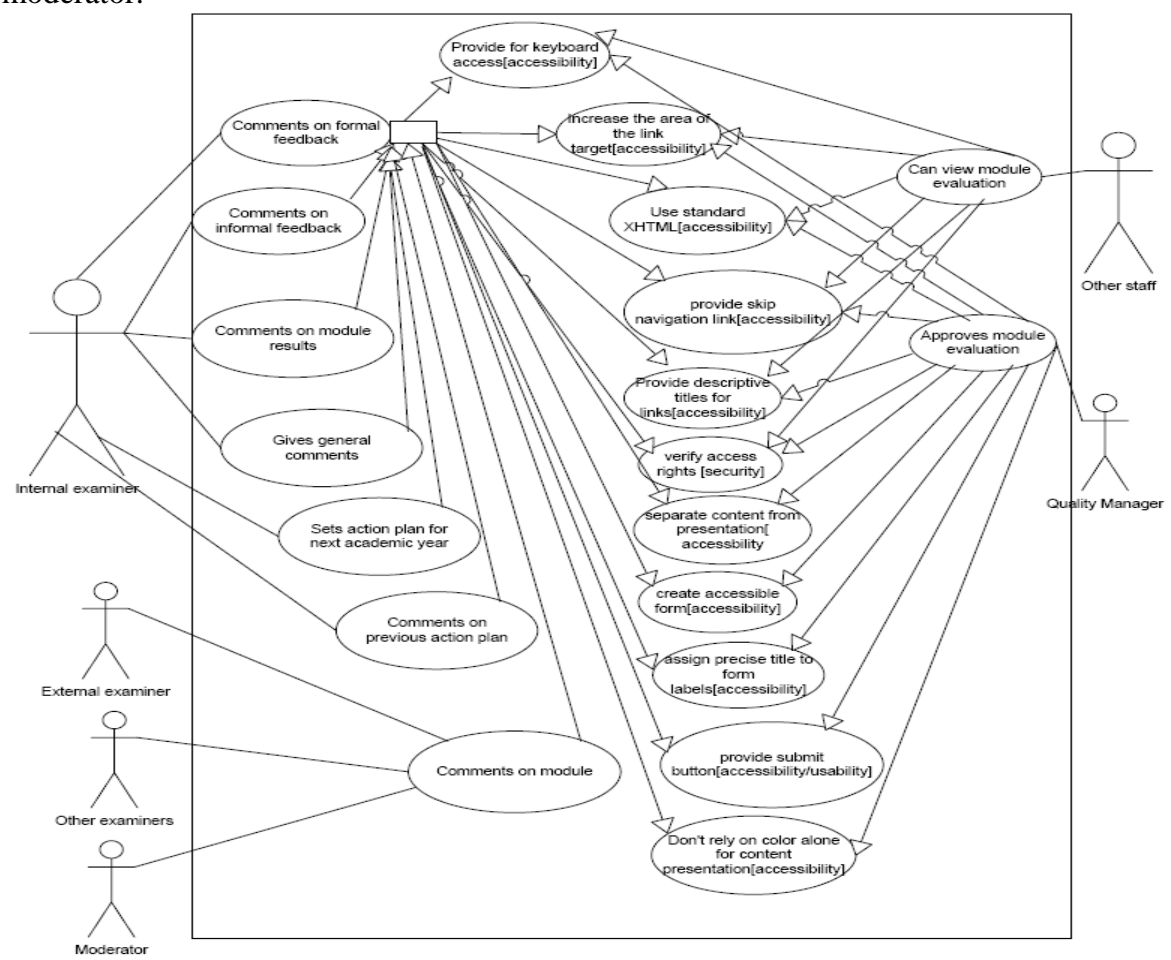

Fig. 2. The use case diagram of the online module review system after required accessibility considerations 


\subsection{Lessons Learnt and Benefits of the Approach}

From the experience of the case study, we observed that Web accessibility can benefit from the approach in a number of ways namely; Early detection of any conflicts between visual design and accessible design e.g. the offline module review system was using color to highlight certain types of content therefore to maintain the format for the sighted but also make it accessible, an alternative format was a necessary web accessibility requirement. Secondly the search for solutions is driven by user needs rather than available/known solutions such as those given in guidelines. In addition, the AR graphs and requirements specification models provide the first basis for the accessibility evaluation of developed system. More so, using the approach guides the selection of the technologies and tools to use e.g. the Quality Manager wanted each section of the review form to behave independently from others. The best technology for this was AJAX. But it is not simple matter to make dynamic (AJAX) behavior accessible. So guidance was sought from ARIA standard [16] and a possible toolscript libraries which have been built with accessibility in mind like JQuery [17] was also earmarked.

Broadly, based on the survey on web developers, the case study and existing literature, the approach provides a number of benefits namely;

- Efficiency: Developers can quickly develop accessibility solutions and spend less time guessing and having to go back to fix problems.

- Effectiveness: The better developers understand the issues, the better they can implement more effective solutions. Henry [12] uses an example of a building architecturally planned for accessibility with a ramp that fits into the building design aesthetically and practically to a building with a ramp added after the building is already designed. The ramp of the later case is likely to look awkward and be less useful to all.

- Less cost: Ensuring that developers understand a wide range of functional limitations during design activities helps avoid costly changes later.

- Without a deliberate process to consider others, it is common for designers to design for their own preferences, abilities and environment [12, 18]. There fore a method guiding developers on how to keep their focus on both accessibility needs and functional needs during web development has a potential to improve the rate of accessibility of web based systems.

\section{Conclusion and Future Work}

Despite the availability of accessibility guidelines, coding and accessibility evaluation tools, the rate of Web accessibility is still disappointing even on websites that have followed the guidelines and or used the tools. To-date, common accessibility problems are caused by focusing on technical aspects at the expense of usability. We have demonstrated an approach to collect, analyze and integrate accessibility needs with functional needs. This approach has a potential to improve accessibility of websites as well as provide other benefits to Web projects. In future, we will test the 
approach on more website projects and Web developers involved in developing accessible websites.

\section{References}

1. Chung and Nixon (1995). Dealing with Non-Functional Requirements: Three Experimental Studies of a Process-Oriented Approach, ICSE '95, Washington USA

2. Chung, Nixon and Yu (1994). Using Quality Requirements to Systematically Develop Quality Software, $4^{\text {th }}$ Intern. Conf. on Software Quality McLean, VA, U.S.A. Oct. 1994.

3. Cysneiros and Leite (2004). Nonfunctional Requirements: From Elicitation to Conceptual Models, IEEE Transactions on Software Engineering, Vol. 30, No. 5, May 2004

4. Huang, C.J. (2003). Usability of E-Government Web Sites for PWDs, In Proc. of the 36th Hawaii Int. Conf. on System Sciences (HICSS’03), IEEE Computer Society, 2003.

5. Nielsen, J. (2001). Beyond Accessibility: Treating Users with Disabilities as People. Jakob Nielsen's Alertbox Nov. 11, 2001. Accessed on 5th November 2006 from: http//www.useit.com /alertbox/20011111.html.

6. Takagi H. et al. (2005). Accessibility Designer: Visualizing Usability for the Blind. ASSETS'04 October 18-20, 2004 Atlanta, Georgia, USA, ACM 2004.

7. Disability Rights Commission (DRC), (2004). Disability Rights Commission. The web access and inclusion for disabled people. Technical report, Disability Rights Commission (DRC), UK, 2004

8. Abanumy et al,. (2005). E-government website Accessibility: In-Depth Evaluation of Saudi Arabia and Oman. The Electronic Journal of e-Government Volume 3 Issue 3.

9. Baguma et al., (2007). Accessibility of E-government Web Sites for people With Disabilities in Uganda", In proc. of the International Computer Science and ICT Conference 5th to 7th February 2007, Nairobi, Kenya.

10. Asakawa (2005). What's the Web Like If You Can't See It?, W4A at WWW2005, 10th May 2005, Chiba, Japan

11. Kelly et al., (2005).Forcing Standardization or Accommodating Diversity? A Framework for Applying the WCAG in the Real World, W4A at WWW2005, 10th May 2005, Chiba, Japan

12. Henry (2007). Just Ask: Integrating Accessibility Throughout Design from http://www.uiaccess.com/accessucd/index.html

13. Mline et al., (2005). Are guidelines enough? An introduction to designing Web sites accessible to older people, IBM Systems Journal, Vol 44, No 3, 2005

14. McEwen, S. (2004). Requirements: An introduction. http://www.ibm.com/developerworks/rational/library/4166.html?S_TACT=105AGX78\&S $\mathrm{CMP}=\mathrm{HP}$

15. Henry, S.L., Martinson, M.L., and Barnicle, K. (2003). Beyond Video: Accessibility Profiles, Personas, and Scenarios Up Close and Personal. Proceedings of UPA 2003 (Usability Professionals' Association annual conference), 2003.

16. http://webaim.org/techniques/aria/ accessed on 2nd February 2009

17. Reagan, B. (200). Accessibility and Design: Failure of the Imagination, W4A at WWW2004, New York, USA. 